\title{
GENERATING FUNCTIONS ON EXTENDED JACOBI POLYNOMIALS FROM LIE GROUP VIEW POINT
}

\author{
Manik Chandra Mukherjee
}

\begin{abstract}
Generating functions play a large role in the study of special functions. The present paper deals with the derivation of some novel generating functions of extended Jacobi polynomials by the application of group-theoretic method introduced by Louis Weisner. In fact, by suitably interpreting the index $(n)$ and the parameter $(\beta)$ of the polynomial under consideration we define four linear partial differential operators and on showing that they generate a Lie-algebra, we obtain a new generating relation (3.3) as the main result of our investigation. Furthermore, some generating functions of Laguerre, Hermite, Bessel and Jacobi polynomials are obtained as the special cases of our main result. Some applications of our results are also pointed out.
\end{abstract}

\section{Introduction}

The extended Jacobi polynomials as defined by I. Fujiwara [1] are as follows:

$$
\begin{aligned}
F_{n}(\alpha, \beta ; x)= & \frac{(-1)^{n}}{n !}\left(\frac{\lambda}{b-a}\right)^{n}(x-a)^{-\alpha}(b-x)^{-\beta} \\
& \times D^{n}\left[(x-a)^{n+\alpha}(b-x)^{n+\beta}\right], \text { where } D \equiv \frac{d}{d x} .
\end{aligned}
$$

They satisfy the following ordinary differential equation:

$$
\begin{aligned}
{\left[(x-a)(b-x) D^{2}+\{(\alpha+1)(b-x)\right.} & -(\beta+1)(x-a)\} D \\
& +n(1+\alpha+\beta+n)] y=0 .
\end{aligned}
$$

Recently, some attempts [2], [3] have been made by researchers for deriving generating functions of the polynomials under consideration from 
the Lie group view point. The aim at presenting this article is to apply L. Weisner's group-theoretic method [4] with the simultaneous suitable interpretations of the index $(n)$ and the parameter $(\beta)$ in the study of extended Jacobi polynomials. It may be mentioned that in the course of constructing a four dimensional Lie algebra we have obtained two operators such that, when operated on the polynomials under consideration, simultaneously raise (lower) and lower (raise) the index and the parameter by one unit. Such type of operators do not seem to have appeared earlier in the study of extended Jacobi polynomials. The main results of this paper are the formulas (3.3) to (3.6) given in Section 3.

\section{Group Theoretic Method}

Replacing $\frac{d}{d x}$ by $\frac{\partial}{\partial x}, n$ by $y \frac{\partial}{\partial y}, \beta$ by $z \frac{\partial}{\partial z}$ and $u$ by $v(x, y, z)$ in $(1.2)$, we get the following partial differential equation:

$$
\begin{aligned}
& (x-a)(b-x) \frac{\partial^{2} v}{\partial x^{2}}+(a-x) z \frac{\partial^{2} v}{\partial x \partial z}+y z \frac{\partial^{2} v}{\partial y \partial z} \\
& +y^{2} \frac{\partial^{2} v}{\partial y^{2}}+\{(1+\alpha)(b-x)-(x-a)\} \frac{\partial v}{\partial x}+(2+\alpha) y \frac{\partial v}{\partial y}=0
\end{aligned}
$$

Thus $v_{1}(x, y, z)=F_{n}(\alpha, \beta ; x) y^{n} z^{\beta}$ is a solution of $(2.1)$ since $F_{n}(\alpha, \beta ; x)$ is a solution of (1.2). Let us now introduce a set of linear partial differential operators, $A_{i}, i=1,2,3,4$, defined as follows:

$$
\left\{\begin{aligned}
A_{1}= & y \frac{\partial}{\partial y} \\
A_{2}= & z \frac{\partial}{\partial z} \\
A_{3}= & \frac{(x-a) y^{-1} z}{\lambda} \frac{\partial}{\partial x}-\frac{z}{\lambda} \frac{\partial}{\partial y} \\
A_{4}= & \frac{\lambda}{b-a}\left[(x-a)(x-b) y z^{-1} \frac{\partial}{\partial x}+(x-b) y^{2} z^{-1} \frac{\partial}{\partial y}\right. \\
& \left.+(x-a) y \frac{\partial}{\partial z}+(1+\alpha)(x-b) y z^{-1}\right]
\end{aligned}\right.
$$

Then

$$
\begin{aligned}
& A_{1}\left(F_{n}(\alpha, \beta ; x) y^{n} z^{\beta}\right)=n F_{n}(\alpha, \beta ; x) y^{n} z^{\beta} \\
& A_{2}\left(F_{n}(\alpha, \beta ; x) y^{n} z^{\beta}\right)=\beta F_{n}(\alpha, \beta ; x) y^{n} z^{\beta} \\
& A_{3}\left(F_{n}(\alpha, \beta ; x) y^{n} z^{\beta}\right)=(n+\alpha) F_{n-1}(\alpha, \beta+1 ; x) y^{n-1} z^{\beta+1} \\
& A_{4}\left(F_{n}(\alpha, \beta ; x) y^{n} z^{\beta}\right)=(n+1) F_{n+1}(\alpha, \beta-1 ; x) y^{n+1} z^{\beta+1} .
\end{aligned}
$$


We now proceed to find the commutator relations satisfied by $A_{i}$ $(i=1,2,3,4)$. Using the notation

$$
[A, B] u=(A B-B A) u
$$

we get

$$
\begin{aligned}
& {\left[A_{1}, A_{2}\right]=0,} \\
& {\left[A_{1}, A_{3}\right]=-A_{3},} \\
& {\left[A_{1}, A_{4}\right]=A_{4},} \\
& {\left[A_{2}, A_{3}\right]=A_{3},} \\
& {\left[A_{2}, A_{4}\right]=-A_{4} \text { and }} \\
& {\left[A_{3}, A_{4}\right]=2 A_{1}+(1+\alpha) .}
\end{aligned}
$$

So from the above commutator relations we can easily state the following:

Theorem. The set of operators $\left\{1, A_{i}(i=1,2,3,4)\right\}$ where 1 stands for the identity operator, generates a Lie-algebra $\mathcal{L}$.

Now the partial differential operator $L$ given by

$$
\begin{aligned}
L= & (x-a)(b-x) \frac{\partial^{2}}{\partial x^{2}}+(a-x) z \frac{\partial^{2}}{\partial x \partial z}+y z \frac{\partial^{2}}{\partial y \partial z} \\
& +y^{2} \frac{\partial^{2}}{\partial y^{2}}+\{(1+\alpha)(b-x)-(x-a)\} \frac{\partial}{\partial x}+(2+\alpha) \frac{\partial}{\partial y}
\end{aligned}
$$

can be expressed as follows:

$$
(x-a) L=(b-a)\left(A_{4} A_{3}+A_{1}^{2}+\alpha A_{1}\right) .
$$

We can easily verify that each of $A_{i}(i=1,2,3,4)$ commutes with $L$. In other words,

$$
\left[(x-a) L, A_{i}\right]=0 .
$$

Now the extended forms of the groups generated by $A_{i}(=1,2,3,4)$ are given as follows:

(2.7) $e^{a_{1} A_{1}} f(x, y, z)=f\left(x, e^{a_{1}} y, z\right)$,

(2.8) $e^{a_{2} A_{2}} f(x, y, z)=f\left(x, y, e^{a_{2}} z\right)$,

$$
e^{a_{3} A_{3}} f(x, y, z)=f\left(\frac{a\left(y-a_{3} \frac{z}{\lambda}\right)+(x-a) y}{y-a_{3} \frac{z}{\lambda}}, y-a_{3} \frac{z}{\lambda}, z\right)
$$

(2.10) $e^{a_{4} A_{4}} f(x, y, z)=\left(1-\frac{\lambda}{b-a}(x-b) a_{4} \frac{y}{z}\right)^{-(1+\alpha)}$

$$
\times f\left(\frac{x-\frac{a \lambda}{b-a}(x-b) a_{4} \frac{y}{z}}{1-\frac{\lambda}{b-a}(x-b) a_{4} \frac{y}{z}}, \frac{y}{1-\frac{\lambda}{b-a}(x-b) a_{4} \frac{y}{z}}, \frac{z\left(1+\lambda a_{4} \frac{y}{z}\right)}{\left(1-\frac{\lambda}{b-a}(x-b) a_{4} \frac{y}{z}\right)}\right) .
$$


Thus we have

$$
\begin{gathered}
e^{a_{4} A_{4}} e^{a_{3} A_{3}} e^{a_{2} A_{2}} e^{a_{1} A_{1}} f(x, y, z)=\left\{1-\frac{\lambda}{b-a}(x-b) a_{4} \frac{y}{z}\right\}^{-(1+\alpha)} \\
\times f\left(\frac{\lambda y\left(x-\frac{a \lambda}{b-a}(x-b) a_{4} \frac{y}{z}\right)-a a_{3} z\left(1+\lambda a_{4} \frac{y}{z}\right)\left(1-\frac{\lambda}{b-a}(x-b) a_{4} \frac{y}{z}\right)}{\left(1-\frac{\lambda}{b-a}(x-b) a_{4} \frac{y}{z}\right)\left(\lambda y-a_{3} z\left(1+\lambda a_{4} \frac{y}{z}\right)\right)},\right. \\
\left.e^{a_{1}}\left(\frac{\lambda y-a_{3} z\left(1+\lambda a_{4} \frac{y}{z}\right)}{\lambda\left(1-\frac{\lambda}{b-a}(x-b) a_{4} \frac{y}{z}\right)}\right), \frac{e^{a_{2}} z\left(1+\lambda a_{4} \frac{y}{z}\right)}{1-\frac{\lambda}{b-a}(x-b) a_{4} \frac{y}{z}}\right) .
\end{gathered}
$$

\section{Generating Functions}

From $(2.1)$ it is seen that $F_{n}(\alpha, \beta ; x) y^{n} z^{\beta}$ is a solution of the system:

$$
\left\{\begin{array}{l}
\left(A_{1}-n\right) u=0 \\
L u=0
\end{array} ;\left\{\begin{array}{l}
\left(A_{2}-\beta\right) u=0 \\
L u=0
\end{array} ;\left\{\begin{array}{l}
\left(A_{1}+A_{2}-n-\beta\right) u=0 \\
L u=0
\end{array} .\right.\right.\right.
$$

From (2.5) we observe that

$$
S(x-a) L\left(F_{n}(\alpha, \beta ; x) y^{n} z^{\beta}\right)=(x-a) L S\left(F_{n}(\alpha, \beta ; x) y^{n} z^{\beta}\right)=0,
$$

where

$$
S=e^{a_{4} A_{4}} e^{a_{3} A_{3}} e^{a_{2} A_{2}} e^{a_{1} A_{1}} .
$$

Therefore, the transformation $S\left(F_{n}(\alpha, \beta ; x) y^{n} z^{\beta}\right)$ is annihilated by $(x-a) L$. Now putting $\alpha_{1}=\alpha_{2}=0$ and replacing $f(x, y, z)$ by $F_{n}(\alpha, \beta ; x) y^{n} z^{\beta}$ we get

$$
\begin{aligned}
e^{a_{4} A_{4}} e^{a_{3} A_{3}}\left(F_{n}(\alpha, \beta ; x) y^{n} z^{\beta}\right) & \left(1+\lambda a_{4} \frac{y}{z}\right)^{\beta} \\
= & \left(1-\frac{\lambda}{b-a}(x-b) a_{4} \frac{y}{z}\right)^{-(1+\alpha+\beta+n)}\left(1-\frac{a_{3} z}{\lambda y}\left(1+\lambda a_{4} \frac{y}{z}\right)\right)^{n} \\
& \times\left(x-\frac{a \lambda}{b-a}(x-b) a_{4} \frac{y}{z}\right)-a a_{3} z\left(1+\lambda a_{4} \frac{y}{z}\right)\left(1-\frac{\lambda}{b-a}(x-b) a_{4} \frac{y}{z}\right) \\
& \quad F_{n}\left(\alpha, \beta, \frac{\lambda y\left(1-\frac{\lambda}{b-a}(x-b) a_{4} \frac{y}{z}\right)\left(\lambda y-a_{3} z\left(1+\lambda a_{4} \frac{y}{z}\right)\right)}{2}\right) .
\end{aligned}
$$


But

$$
\begin{aligned}
& e^{a_{4} A_{4}} e^{a_{3} A_{3}}\left(F_{n}(\alpha, \beta ; x) y^{n} z^{\beta}\right) \\
& =\sum_{k=0}^{\infty} \sum_{p=0}^{n+k} \frac{\left(a_{3}\right)^{p}}{p !} \frac{\left(a_{4}\right)^{k}}{k !}(-1)^{p}(-n-\alpha)_{p}(n-p+1)_{k} \\
& \quad \times F_{n-p+k}(\alpha, \beta+p-k ; x) y^{n-p+k} z^{\beta+p-k} .
\end{aligned}
$$

Equating (3.1) and (3.2) we get our main result:

$$
\begin{aligned}
& \left(1-\frac{\lambda}{b-e}(x-b) a_{4} \frac{y}{z}\right)^{-(1+\alpha+\beta+n)}\left(1+\lambda a_{4} \frac{y}{z}\right)^{\beta} \\
& \times\left(1-\frac{a_{3} z}{\lambda y}\left(1+\lambda a_{4} \frac{y}{z}\right)\right)^{n} \\
& F_{n}\left(\alpha, \beta, \frac{\lambda y\left(x-\frac{a \lambda}{b-a}(x-b) a_{4} \frac{y}{z}\right)-a a_{3} z\left(1+\lambda a_{4} \frac{y}{z}\right)\left(1-\frac{\lambda}{b-a}(x-b) a_{4} \frac{y}{z}\right)}{\left(1-\frac{\lambda}{b-a}(x-b) a_{4} \frac{y}{z}\right)\left(\lambda y-a_{3} z\left(1+\lambda a_{4} \frac{y}{z}\right)\right)}\right) \\
& =\sum_{k=0}^{\infty} \sum_{p=0}^{n+k} \frac{\left(a_{3}\right)^{p}}{p !} \frac{\left(a_{4}\right)^{k}}{k !}(-1)^{p}(-n-\alpha)_{p}(n-p+1)_{k} \\
& \quad \times F_{n-p+k}(\alpha, \beta+p-k ; x) y^{n-p+k} z^{\beta+p-k} .
\end{aligned}
$$

Before discussing the particular cases of (3.3) we would like to point out that the operators $A_{3}, A_{4}$ being non commutative, the relation (3.3) will change if we change the order of the Lie element $e^{a_{4} A_{4}} e^{a_{3} A_{3}}$. This is done in Section 4.

Now we discuss several cases:

Case 1. Putting $a_{4}=0, a_{3}=1$ and $-\frac{z}{y}=t$ in (3.3) we get

$$
\begin{aligned}
\left(1+\frac{t}{\lambda}\right)^{n} F_{n}\left(\alpha, \beta ; \frac{x+\frac{a t}{\lambda}}{1+\frac{t}{\lambda}}\right) & \\
& =\sum_{p=0}^{\infty} \frac{(-n-\alpha)_{p}}{p !} F_{n-p}(\alpha, \beta+p ; x) t^{p}
\end{aligned}
$$


Case 2. Putting $a_{3}=0, a_{4}=1$ and $\frac{y}{z}=t$ in (3.3) we get

$$
\begin{array}{r}
\left(1-\frac{\lambda}{b-a}(x-b) t\right)^{-(1+\alpha+\beta+n)}(1+\lambda t)^{\beta} \times F_{n}\left(\alpha, \beta, \frac{x-\frac{a \lambda}{b-a}(x-b) t}{1-\frac{\lambda}{b-a}(x-b) t}\right) \\
=\sum_{k=0}^{\infty} \frac{(n+1)_{k}}{k !} F_{n+k}(\alpha, \beta-k ; x) t^{k}
\end{array}
$$

Case 3. Putting $a_{3}=1, a_{4}=\frac{1}{w}$ and $\frac{y}{z}=t$ in (3.3) we get

$$
\begin{gathered}
\left(1-\frac{\lambda}{b-a}(x-b) \frac{t}{w}\right)^{-(1+\alpha+\beta+n)}\left(1+\frac{\lambda t}{w}\right)^{\beta}\left(1-\frac{1}{\lambda t}\left(1+\frac{\lambda t}{w}\right)\right)^{n} \\
\times F_{n}\left(\alpha, \beta, \frac{\lambda y\left(x-\frac{a \lambda}{b-a}(x-b) \frac{t}{w}\right)-a z\left(1+\frac{\lambda t}{w}\right)\left(1-\frac{\lambda}{b-a}(x-b) \frac{t}{w}\right)}{\left(1-\frac{\lambda}{b-a}(x-b) \frac{t}{w}\right)\left(\lambda y-z\left(1+\frac{t}{w}\right)\right)}\right) \\
=\sum_{k=0}^{\infty} \sum_{p=0}^{n+k} \frac{1}{w^{k}} \frac{1}{k !} \frac{(-1)^{p}(-n-\alpha)_{p}}{p !}(n-p+1)_{k} \\
\times F_{n-p+k}(\alpha, \beta+p-k ; x) t^{k-p} .
\end{gathered}
$$

We now proceed to find some particular cases of interest of results (3.4) and (3.5).

\section{Particular Case 1: (On Jacobi Polynomials).}

Putting $-a=b=1$ and $\lambda=1$ in (3.4) and (3.5) we get the following generating relations of Jacobi polynomials $[8]$

$$
(1+t)^{n} p_{n}^{(\beta, \alpha)}\left(\frac{x-t}{1+t}\right)=\sum_{p=0}^{\infty} \frac{(-n-\alpha)_{p}}{p !} p_{n-p}^{(\beta+p, \alpha)}(x) t^{p}
$$

and

$$
\begin{aligned}
& \text { (3.8) }\left(1-\frac{t}{2}(x-1)\right)^{-(1+\alpha+\beta+n)}(1+t)^{\beta} p_{n}^{(\beta, \alpha)}\left(\frac{x+\frac{t}{2}(x-1)}{1-\frac{t}{2}(x-1)}\right) \\
& =\sum_{k=0}^{\infty} \frac{(n+1)_{k}}{k !} p_{n+k}^{(\beta-k, \alpha)}(x) t^{k} \text {. }
\end{aligned}
$$


ON GENERATING FUNCTIONS OF EXTENDED JACOBI POLYNOMIALS 9

Now by using the symmetry relation

$$
p_{n}^{(\alpha, \beta)}(-x)=(-1)^{n} p_{n}^{(\beta, \alpha)}(x)
$$

we get the following generating relation $[\mathbf{9}]$

$$
(1+t)^{n} p_{n}^{(\alpha, \beta)}\left(\frac{x-t}{1+t}\right)=\sum_{p=0}^{\infty} \frac{(-n-\alpha)_{p}}{p !} p_{n-p}^{(\alpha+p, \beta)}(x) t^{p}
$$

and

$$
\begin{array}{r}
(1+t)^{\alpha}\left(1-\frac{t}{2}(x-1)\right)^{-(1+\alpha+\beta+n)} \\
p_{n}^{(\alpha, \beta)}\left(\frac{x-\frac{t}{2}(x-1)}{1-\frac{t}{2}(x-1)}\right) \\
=\sum_{k=0}^{\infty} \frac{(n+1)_{k}}{k !} p_{n+k}^{(\alpha-k, \beta)}(x) t^{k}
\end{array}
$$

Particular Case 2: (On Laguerre Polynomials).

Putting $a=0, \lambda=1, \beta=b$ and taking limit as $\beta \rightarrow \infty$, we get the following generating relations of Laguerre polynomials $[\mathbf{6}]$

$$
\begin{aligned}
(1-t)^{-1-\alpha-n} \exp \left(-\left(\frac{t x}{1-t}\right)\right) L_{n}^{(\alpha)} & \left(\frac{x}{1-t}\right) \\
& =\sum_{k=0}^{\infty} \frac{(n+1)_{k}}{k !} L_{n+k}^{(\alpha)}(x) t^{k}
\end{aligned}
$$

and

$$
(1-t)^{n} L_{n}^{(\alpha)}\left(\frac{x}{1-t}\right)=\sum_{p=0}^{\infty} \frac{(-n-\alpha)_{p}}{p !} L_{n-p}^{(\alpha)}(x) t^{p} .
$$

Particular Case 3: (On Hermite Polynomials).

Putting $\alpha=\beta,-a=b=\sqrt{a}, \lambda=\frac{2}{\sqrt{\alpha}}$ and taking limit as $\alpha \rightarrow \infty$, we get the following generating relations of Hermite polynomials [5]

$$
H_{n}(x+y)=\sum_{p=0}^{n}\left(\begin{array}{l}
n \\
p
\end{array}\right) H_{n-p}(x)(2 y)^{p}
$$

and

$$
e^{2 x t-t^{2}} H_{n}(x-t)=\sum_{k=0}^{\infty} \frac{1}{k !} H_{n+k}(x) t^{k} .
$$


Particular Case 4: (On Bessel Polynomials).

Replacing $x$ by $1+\frac{2 x \varepsilon}{s}, t$ by $\frac{s w}{\varepsilon}$, putting $-a=b=\lambda=1, \alpha=v-\varepsilon-1$, $\beta=\varepsilon-1$ and then taking the limit as $\varepsilon \rightarrow \infty$, we get the following generating relations of Bessel's polynomials [7]

$$
\begin{aligned}
e^{s w}(1-x w)^{1-\nu-n} Y_{n}\left(\frac{x}{1-x w} ; \nu, s\right) & \\
& =\sum_{k=0}^{\infty} e^{k} Y_{n+k}(x ; \nu-k, s) \frac{w^{k}}{k !}
\end{aligned}
$$

and

$$
(1+t)^{n} Y_{n}\left(\frac{w}{1+t} ; \nu, s\right)=\sum_{p=0}^{n}\left(\begin{array}{l}
n \\
p
\end{array}\right) Y_{n-p}(x ; \nu+p, s) t^{p}
$$

\section{Variants of the Result (3.3)}

Since $\left[A_{3}, A_{4}\right] \neq 0$, we can well aply the operator $e^{a_{3} A_{3}} \times e^{a_{4} A_{4}}$ on the function $F_{n}(\alpha, \beta ; x) y^{n} z^{\beta}$.

Now

$$
\begin{aligned}
& e^{a_{3} A_{3}} e^{a_{4} A_{4}}\left(F_{n}(\alpha, \beta ; x) y^{n} z^{\beta}\right) \\
& =\left\{1-a_{4}\left(a_{3}+\frac{\lambda}{b-a}(x-b) \frac{y}{z}\right)\right\}^{-(1+\alpha+\beta+n)}\left(1-\frac{a_{3} z}{\lambda y}\right)^{n} \\
& \quad \times\left(1+\frac{a_{4}}{z}\left(\lambda y-a_{3} z\right)\right)^{\beta} \\
& \quad \times F_{n}\left(\frac{\left(x y-\frac{a a_{3} z}{\lambda}\right)-a a_{4}\left(y-\frac{a_{3} z}{\lambda}\right)\left(a_{3}+\frac{\lambda}{b-a}(x-b) \frac{y}{z}\right)}{\left(y-\frac{a_{3} z}{\lambda}\right)\left(1-a_{4}\left(a_{3}+\frac{\lambda}{b-a}(x-b) \frac{y}{z}\right)\right)}\right) .
\end{aligned}
$$

Again

$$
\begin{aligned}
& e^{a_{3} A_{3}} e^{a_{4} A_{4}}\left(F_{n}(\alpha, \beta ; x) y^{n} z^{\beta}\right) \\
& =\sum_{k=0}^{\infty} \sum_{p=0}^{n+k} \frac{\left(a_{3}\right)_{p}}{p !} \frac{\left(a_{4}\right)^{k}}{k !}(-1)^{p}(-\alpha-n-k)_{p}(n+1)_{k} \\
& \quad \times F_{n+k-p}(\alpha, \beta-k+p ; x)\left(\frac{y}{z}\right)^{k-p} .
\end{aligned}
$$


ON GENERATING FUNCTIONS OF EXTENDED JACOBI POLYNOMIALS 11

Equating (4.1) and (4.2) we get the following result

$$
\begin{aligned}
& \left\{1-a_{4}\left(a_{3}+\frac{\lambda}{b-a}(x-b) \frac{y}{z}\right)\right\}^{-(1+\alpha+\beta+n)}\left(1-\frac{a_{3} z}{\lambda y}\right)^{n} \\
& \quad \times\left(1+\frac{a_{4}}{z}\left(\lambda y-a_{3} z\right)\right)^{\beta} \\
& \quad \times F_{n}\left(\frac{\left(x y-\frac{a a_{3} z}{\lambda}\right)-a a_{4}\left(y-\frac{a_{3} z}{\lambda}\right)\left(a_{3}+\frac{\lambda}{b-a}(x-b) \frac{y}{z}\right)}{\left(y-\frac{a_{3} z}{\lambda}\right)\left(1-a_{4}\left(a_{3}+\frac{\lambda}{b-a}(x-b) \frac{y}{z}\right)\right)}\right) \\
& =\sum_{k=0}^{\infty} \sum_{p=0}^{n+k} \frac{\left(a_{3}\right)_{p}}{p !} \frac{\left(a_{4}\right)^{k}}{k !}(-1)^{p}(-\alpha-n-k)_{p}(n+1)_{k} \\
& \quad \times F_{n+k-p}(\alpha, \beta-k+p ; x)\left(\frac{y}{z}\right)^{k-p} .
\end{aligned}
$$

\section{Application}

Relations (3.4) and (3.5) may be applied in deriving bilateral generating functions involving the special function under consideration. We shall give an application by using the relation (3.5) in deriving the following theorem as bilateral generating relation.

Theorem. If

$$
G(x, w)=\sum_{n=0}^{\infty} a_{n} F_{n}(\alpha, \beta ; x) w^{n}
$$

then

$$
\begin{aligned}
(1+\lambda t)^{\beta}\{1 & \left.-\frac{\lambda}{b-a}(x-b) t\right\}^{-(1+\alpha+\beta)} \\
& \times G\left(\frac{x-\frac{\lambda a}{b-a}(x-b) t}{1-\frac{\lambda}{b-a}(x-b) t}, \frac{z t}{1-\frac{\lambda}{b-a}(x-b) t}\right) \\
= & \sum_{n=0}^{\infty} t^{n} \sigma_{n}(x, z)
\end{aligned}
$$

where

$$
\sigma_{n}(x, z)=\sum_{k=0}^{\infty} a_{k} \frac{(k+1)_{n-k}}{(n-k) !} F_{n}(\alpha, \beta-n+k ; x) z^{k}
$$


Proof:

$$
\begin{aligned}
& \sum_{n=0}^{\infty} t^{n} \sigma_{n}(x, z) \\
& =\sum_{n=0}^{\infty} t^{n} \sum_{k=0}^{n} a_{k} \frac{(k+1)_{n-k}}{(n-k) !} F_{n}(\alpha, \beta-n+k ; x) z^{k} \\
& =\sum_{n=0}^{\infty} \sum_{k=0}^{\infty} a_{k} \frac{(k+1)_{n}}{n !} F_{n+k}(\alpha, \beta-n ; x) z^{k} t^{n+k} \\
& =\sum_{k=0}^{\infty} a_{k}(z t)^{k} \sum_{n=0}^{\infty} \frac{(k+1)_{n}}{n !} F_{n+k}(\alpha, \beta-n ; x) t^{n} \\
& =\sum_{k=0}^{\infty} a_{k}(z t)^{k}\left\{1-\frac{\lambda}{b-a}(x-b) t\right\}^{-(1+\alpha+\beta+k)}(1+\lambda t)^{\beta} \\
& \times F_{n}\left(\alpha, \beta, \frac{x-\frac{\lambda a}{b-a}(x-b) t}{1-\frac{\lambda}{b-a}(x-b) t}\right) \\
& =\left\{1-\frac{\lambda}{b-a}(x-b) t\right\}^{-(1+\alpha+\beta)}(1+\lambda t)^{\beta} \\
& \times \sum_{k=0}^{\infty} a_{k}(z t)^{k} F_{n}\left(\alpha, \beta ; \frac{x-\frac{\lambda}{b-a}(x-b) t}{1-\frac{\lambda}{b-a}(x-b) t}\right) \frac{1}{\left(1-\frac{\lambda}{b-a}(x-b) t\right)^{k}} \\
& =\left\{1-\frac{\lambda}{b-a}(x-b) t\right\}^{-(1+\alpha+\beta)}(1+\lambda t)^{\beta} \\
& \times G\left(\frac{x-\frac{\lambda a}{b-a}(x-b) t}{1-\frac{\lambda}{b-a}(x-b) t}, \frac{z t}{1-\frac{\lambda}{b-a}(x-b) t}\right) .
\end{aligned}
$$

The importance of this theorem lies in the fact that one can get a large number of bilateral generating relations from (ii) by attributing different suitable values to $a_{n}$ in (i).

\section{References}

1. I. FuJIWARA, A unified presentation of classical orthogonal polynomials, Math. Japan 11 (1966), 133-148.

2. P. N. SRivastava And S. S. Dhillon, Lie operator and classical orthogonal polynomials II, Pure Math. Manuscript 7 (1988), 129-136. 
3. M. C. Mukherjee And A. K. Chongdar, Derivation of some generating functions of modified Bessel polynomials by Lie algebraic method, Bull. Calcutta Math. Soc. 80 (1988), 411-416.

4. L. WEISNER, Group-theoretic origin of certain generating functions, Pacific J. Math. 5 (1955), 1033-39.

5. L. WeISNER, Generating functions for Hermite functions, Canad. J. Math. 11 (1959), 141-147.

6. E. B. MCBRIDE, "Obtaining generating functions," Springer Verlag, Berlin, Heidelberg, New York, 1971.

7. A. K. ChOngdar, Some generating functions involving generalised Bessel polynomials, Bull. Calcutta Math. Soc. 77 (1985), 330-339.

8. A. B. Chakrabarti, A. B., Group-theoretic study of certain generalised functions of Jacobi polynomials, J. Indian Inst. Sci. 64(B) (1983), 97-103.

9. B. Ghosh, Group-theoretic origin of certain generating functions of Jacobi polynomials II, Pure Math. Manuscript 5 (1986).

Keywords. Generating functions, Jacobi, Hermite, Laguerre and Bessel polynomials. 1991 Mathematics subject classifications: 33A65.

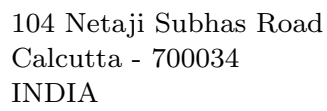

Primera versió rebuda el 27 de Maig de 1993, darrera versió rebuda el 8 de Setembre de 1994 